\title{
THE INCIDENCE OF DEEP VEIN THROMBOSIS AFTER CEMENTLESS AND CEMENTED KNEE REPLACEMENT
}

\author{
YOUNG-HOO KIM
}

From the Yonsei University College of Medicine, Seoul

\begin{abstract}
The incidence of deep vein thrombosis in 244 patients who had total knee replacement has been studied. In 120 the prosthesis was cemented and in 124 it was cementless. In all cases the replacement was primary and a porous-coated prosthesis with a porous-coated central tibial stem was used. Deep vein thrombosis was diagnosed by venography, and pulmonary embolism by perfusion scanning.

The incidence of deep vein thrombosis in the cementless knees $(23.8 \%)$ and in the cemented $(25 \%)$ was approximately the same. The only significant predisposing factors for deep vein thrombosis in both groups were obesity, prolonged postoperative immobilisation, previous venous disease and hyperlipidaemia.
\end{abstract}

The reported incidence of deep vein thrombosis after cemented total knee replacement ranges from $50 \%$ to $72 \%$ (McKenna et al 1976; Lotke et al 1984; Stulberg et al 1984). Clinical risk factors for deep vein thrombosis that have been identified include advanced age, sedentary patients, previous venous thrombo-embolism, the presence of malignant disease, cardiac failure, prolonged immobility or paralysis, obesity, varicose veins, oestrogen therapy, and genetic and dietary factors (Nicolaides and Irving 1975; Kim and Suh 1988). Although cementless knee replacement is performed with increasing frequency in patients with advanced arthritis, the incidence of deep vein thrombosis after this procedure has not been documented.

In 244 consecutive patients undergoing primary total knee replacement venography was performed, both pre-operatively and postoperatively, in an attempt to determine the incidence of deep vein thrombosis as well as its location and the risk factors. The incidence of deep vein thrombosis in cementless as distinct from cemented replacement was also studied.

\section{MATERIALS AND METHODS}

In all, 244 consecutive patients over 50 years of age were studied. They had had primary total knee replacement, using a porous-coated prosthesis with a porous-coated central tibial stem (PCA; Howmedica, Rutherford, New

Y.-H. Kim, MD, Associate Professor

Department of Orthopaedic Surgery, Joint Reconstruction and Implant Service, Texas Tech University School of Medicine, Lubbock, Texas 79430, USA.

(C) 1990 British Editorial Society of Bone and Joint Surgery $0301-620 \mathrm{X} / 90 / 5161 \$ 2.00$

J Bone Joint Surg [Br] 1990; 72-B:779-83.
Jersey). Of these, 124 patients ( 168 knees) had cementless replacement and 120 (160 knees) were cemented. All the patients were requested to discontinue all aspirin or aspirin-containing compounds and any other antiplatelet medication 14 days before admission to hospital for operation.

In the cementless group, 80 patients had unilateral replacements ( 40 left and 40 right), and 44 had bilateral replacements ( 24 were one-staged and 20 two-staged). There were 70 women and 54 men with an average age of 60.5 years (range 50 to 82): 66 were 50 to 59,44 were 60 to 69,12 were 70 to 79 , and two were 80 to 89 . The diagnosis was osteoarthritis in 70 patients ( 94 knees), rheumatoid arthritis in 28 (44), tuberculous arthritis in 18 (20), traumatic arthritis in six (6), and avascular necrosis of the lateral femoral condyle in two (4).

In the cemented group, 80 patients had unilateral replacement and 40 bilateral (18 were one-staged and 22 were two-staged). In 64 patients the right knee was replaced; in 56 the left. There were 70 women and 50 men with an average age of 60.3 years (range 50 to 85 ): 56 were aged 50 to 59,44 were aged 60 to 69,12 were aged 70 to 79 , and eight were between 80 and 89 . The diagnosis was. osteoarthritis in 74 patients (102 knees), rheumatoid arthritis in 42 (54), and tuberculous arthritis in four (4). All patients were followed for at least one year after the arthroplasty.

During the operation (both cementless and cemented), a pneumatic tourniquet was maintained on the proximal part of the thigh to occlude the arterial circulation, and the tourniquet time was recorded. All operations were carried out by the author. No patient wore elastic stockings or elastic wraps either before or during the operation, and postoperatively only 28 patients wore an elastic stocking, which was prescribed because 
of swelling of the lower limb. Two suction drains were placed in the wound; they were removed after 24 hours.

Postoperatively, the limb was immobilised in a cylinder cast splint and elevated on two pillows. Quadriceps exercises were initiated the day after the surgery and all splints and dressings were removed at 48 hours. On the third postoperative day, knee movements were started. A sling was used at all times to encourage flexion by gravity. Standing was allowed on the second postoperative day, and walking with crutches and partial weight-bearing was permitted shortly thereafter. No patient received prophylactic agents for deep vein thrombosis.

Coagulation assays (platelet count, prothrombin time, partial thromboplastin time, fibrinogen, antithrombin III and factor VIII), complete blood count, blood typing and serum chemical profile tests were performed in all patients on three separate occasions: one on the day before operation, one on the second or third postoperative day, and one on the seventh or eighth postoperative day.

The pre-operative venograms were performed bilaterally within two days of the anticipated surgery, and the postoperative venograms at 10 to 11 days. The criterion for diagnosing acute postoperative deep vein thrombosis was a filling defect in a deep vein, or defects surrounded by a narrow rim of contrast material.

Variations of the anatomy of the superficial femoral and popliteal veins were also studied, and the number of valves in the deep veins from the level of the popliteal fossa up to the ischial spine region was tabulated (Fig. 1). The incidence of deep vein thrombosis was correlated with these two factors.

In all cases, electrocardiograms, chest radiographs and serial determinations of blood gases and serum enzymes were performed. All patients had perfusion lung-scanning using a standardised technique. The preoperative examination was compared with that performed nine to 10 days after the arthroplasty. Ventilation lung-scanning and pulmonary angiography were performed if the perfusion lung-scanning was positive; if it was negative, no further studies were done.

The blood loss at operation was assessed by weighing the swabs, calculating the amount of contained blood and adding this to the amount obtained from wound suction. The total volume obtained by suction drainage from the operation site was taken as the postoperative blood loss. The amounts of blood and blood products or blood substitutes used intra-operatively and postoperatively were recorded.

\section{RESULTS}

In neither the cemented nor the uncemented knees were any fresh or old thrombi detected in the pre-operative venograms. Postoperatively, of the 168 venograms in the 124 patients with a cementless replacement, $40(23.8 \%)$
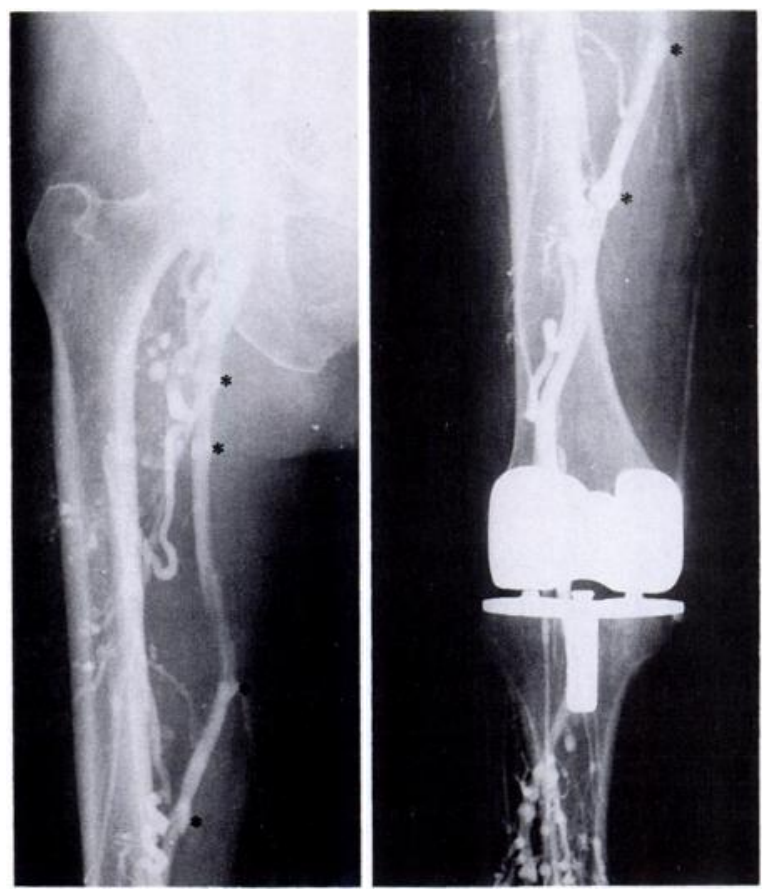

Fig. 1

The number of valves in the superficial femoral vein from the level of the popliteal fossa up to the ischial spine region was counted. In this patient there are six venous valves.

were positive for fresh deep vein thrombosis, while 40 $(25 \%)$ of the 160 venograms were positive in the 120 patients with a cemented replacement. In the two groups all but two thrombi were located in the calf veins; two extended proximally into the popliteal vein (Fig. 2).

In the cementless group, the mean age of the 40 patients with deep vein thrombosis was 63.2 years (range 51 to 69) while the mean age of the 84 patients without deep vein thrombosis was 60.1 years (range 50 to 82 ); 22 of the patients with thrombi were 50 to 59 years old, and 18 were 60 to 69 .

In the cemented group, the mean age of the 40 patients with thrombi was 65 years (range 50 to 75 ), while that of the 80 patients without thrombi was 63.7 years (range 50 to 85); 20 of the patients with thrombi were 50 to 59 years old, 14 were 60 to 69 , and six were aged 70 to 79.

Among the patients with deep vein thrombosis after cementless replacement, 24 knees had osteoarthritis, 12 had rheumatoid arthritis and four had tuberculous arthritis. Of the 70 patients ( 94 knees) with osteoarthritis $24(25.5 \%)$ had deep vein thrombosis, while of the 28 patients (44 knees) with rheumatoid arthritis $12(27.3 \%)$ had deep vein thrombosis. In the cemented group, 26 knees $(25.5 \%)$ in the 74 patients (102 knees) with osteoarthritis and 14 knees $(25.9 \%)$ in the 42 patients ( 54 knees) with rheumatoid arthritis had deep vein thrombosis.

In both cementless and cemented groups, the incidence of deep vein thrombosis after bilateral replace- 


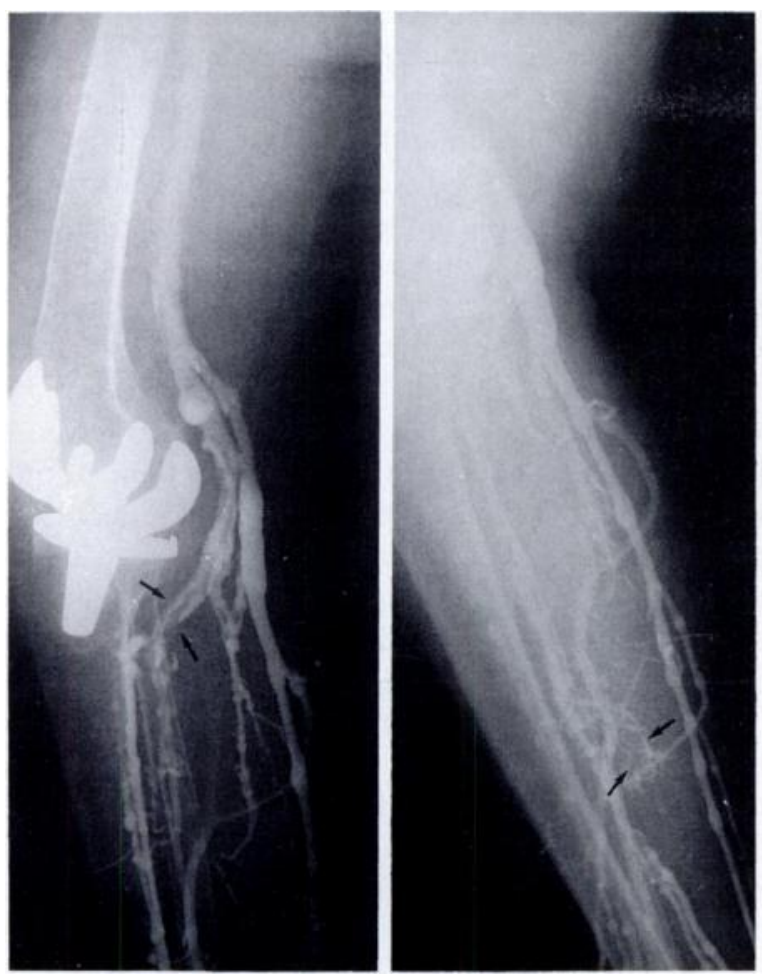

Fig. 2

A 60-year-old woman who had a cementless total knee replacement has deep vein thrombosis in the calf vein, which extends proximally into the popliteal vein.

ment was similar to that after unilateral replacement; furthermore, one-staged bilateral replacement did not increase the risk of developing deep vein thrombosis.

No statistical difference $(p>0.05)$ in the incidence of deep vein thrombosis with respect to venous anatomical variations was found in either the cementless or the cemented knees; nor was there any correlation $(p>0.05)$ between the incidence of deep vein thrombosis and the number of valves in the two groups. In both the cementless and cemented groups, comparison of the coagulation assay data of the normal and the abnormal venograms showed no significant differences pre- or postoperatively.

In the cementless group, the average weight of the patients who did not have deep vein thrombosis was $57.4 \mathrm{~kg}$ (range 44 to 69 ); that of the patients who had thrombosis was $71.7 \mathrm{~kg}$ (range 60 to 89 ). In the cemented group, the average weight of the patients who did not have thrombosis was $55.3 \mathrm{~kg}$ (range 34.5 to 58), while that of the patients who had thrombosis was $68.3 \mathrm{~kg}$ (range 62 to 75 ).

Of the 124 patients with cementless replacement, 24 required treatment for hypertension; four of these patients had thrombosis, but the remaining 20 did not. Of the 120 patients with cemented replacement 14 required treatment for hypertension, but none of them had thrombosis.

In both groups, the average times of anaesthesia, operation and tourniquet in the patients with or without thrombi were not statistically different $(p>0.05)$. In the patients with or without deep vein thrombosis, in both cementless and cemented groups, the blood loss was nearly the same; nor was there any difference in the haematocrit values, whether pre-operative, postoperative on the day of operation, on the first postoperative day, or on the following day. Also, no differences were observed in the pre-operative cholesterol, triglyceride, total protein and calcium levels.

The average intervals from the time of the surgery until the patient sat on the edge of the bed (one day), walked for the first time (three days), and was discharged from hospital (14 days) were the same in the patients with or without thrombosis in both cementless and cemented groups.

No pulmonary emboli occurred in our series, as evidenced by the perfusion lung-scanning and the complete absence of clinical symptoms. One patient had wound separation which required secondary closure. No patient had an allergic reaction to the contrast media used for the venograms.

\section{DISCUSSION}

In this study it emerged that some of the factors widely believed to be associated with deep vein thrombosis did not appear to be so, while other factors did. Despite the common belief, none of the following factors were found to be associated with deep vein thrombosis: advanced age, the underlying disease, one-staged or two-staged bilateral replacement, venous anatomical variations, the number of venous valves, the coagulation assay data, blood group, hypertension, tourniquet time, choice of cemented or cementless replacement, the severity or duration of the operation, profuse operative blood loss, or a large amount of blood transfused.

While several studies have shown that the incidence of deep vein thrombosis and pulmonary embolism increases after 40 years of age (Salzman and Harris 1976; Nillius and Nylander 1979; Sikorski, Hampson and Staddon 1981; Hull and Raskob 1986) others have reported no correlation between the age of the patient and the development of deep vein thrombosis (Cohen et al 1973; McKenna et al 1976; Stulberg et al 1984; Kim and Suh 1988). This present study supports the view that advanced age does not constitute a risk factor.

McKenna et al (1976) claimed that there was a lower incidence of deep vein thrombosis in rheumatoid patients because they had had a high dosage of aspirin in one form or another; and Sikorski et al (1981) reported a higher incidence in patients with osteoarthritis. However, others (Cohen et al 1973; Stulberg et al 1984; Kim and Suh 1988) have reported no such correlation, and this present study supports the view that there is no specific correlation between the different types of arthritis and deep vein thrombosis.

Stulberg et al (1984) reported that while the incidence 
of deep vein thrombosis in each knee after two-staged bilateral total knee replacement was comparable with that after the unilateral replacement, one-staged bilateral replacement substantially increased the risk of developing deep vein thrombosis. Morrey et al (1987) found that the incidence of deep vein thrombosis was no greater in their patients who had two-staged bilateral knee replacement than in those who had unilateral replacement. This present study has shown that the incidence of deep vein thrombosis has no specific correlation with one- or twostaged bilateral replacement. Liu et al (1986) suggested that limbs with multiple superficial femoral veins and with more than five valves were at higher risk of deep vein thrombosis after cemented total hip replacement and claimed that venous stasis may predispose the limb to thrombosis. Kim and Suh (1988) concluded that there was little correlation between venous stasis and the development of thrombosis after cementless total hip replacement.

No significant difference in predilection for thrombosis between patients with anatomical venous variations and those without such anomalies, has been found in the present study, nor between patients with more than five valves and those with less. These findings lead us to believe that there is no correlation between venous stasis and the development of thrombosis.

Stulberg, Lucas and Belhobek (1984) suggested that there was a possible correlation between antithrombin III activity and the occurrence of deep vein thrombosis after a cemented total hip replacement. In their prospective evaluation of antithrombin III activity, they noted abnormal depression of this activity in all patients with documented deep vein thrombosis. Gallus (1981) and Kim and Suh (1988), however, found no such correlation and this present series supports that conclusion.

After a cemented total hip replacement, patients with blood group $\mathrm{O}$ are claimed to have a lower prevalence of deep vein thrombosis than patients with other blood groups (Jick et al 1969; Salvati and Lachiewicz 1976). In this present knee series, patients with group $O$ blood were not necessarily protected from deep vein thrombosis, which is consistent with the finding of Kim and Suh (1988) after cementless hip replacement.

Evarts and Feil (1971) reported that after cemented total hip replacement, patients with hypertension had a higher incidence of deep vein thrombosis. Stulberg et al (1984) reported that after a cemented total knee replacement hypertension was associated with an increased incidence of postoperative thrombosis in the calf but not with the development of thrombosis in the popliteal or thigh veins. This present study found no evidence that hypertension was a contributing factor, and this is consistent with the finding of Kim and Suh (1988) after cementless total hip replacement.

There are controversies regarding the effect of a tourniquet on the development of deep vein thrombosis. McKenna et al (1976) claimed that the local mechanical effect of a tourniquet at the groin and the prolonged ischaemia during total knee replacement might well cause damage to the vessel wall and endothelium, which could lead to adhesion and the aggregation of platelets. Fahmy and Patel (1981), however, observed that release of the pneumatic tourniquet was followed by increased fibrinolytic activity, and concluded that this might be responsible, in part, for the decreased incidence of deep vein thrombosis in patients in whom a pneumatic tourniquet was employed. The present series agrees with the reports by some authors (Cohen et al 1973; Simon et al 1982; Stulberg et al 1984) that there is no relationship between the tourniquet time and the subsequent development of deep vein thrombosis. Nor was prolonged duration of the operation, profuse operative blood loss, nor a large amount of blood transfused associated with a high prevalence of deep vein thrombosis.

There is also controversy regarding the effect of the heat generated by acrylic cement in the formation of deep vein thrombosis. To assess this possibility, we sought to determine whether other risk factors were present equally among cemented and cementless knee replacements; it was found that the use of acrylic cement had no correlation with development of deep vein thrombosis.

However, analysis of our figures suggests that some factors are relevant. Thus, we agree with Atik, Harkess and Wichman (1970), Stulberg et al (1984) and Hull and Raskob (1986), that obesity is associated with a high incidence of deep vein thrombosis in operated and nonoperated legs.

Many authors have postulated that slowness of venous return from the lower extremity may lead to the development of deep vein thrombosis. When the lower limbs are horizontal there is considerable stasis in the veins of the calf. Such stasis is increased further during the operation (McLachlin et al 1960; Doran, Drury and Sivyer 1964; Clark and Cotton 1968; Lewis, Mueller and Edward 1972; Nicolaides et al 1972). It has therefore been claimed that prolonged postoperative immobilisation increases the incidence of deep vein thrombosis, while early mobilisation reduces it (Wright, Osborn and Edmonds 1951; Sevitt and Gallagher 1961; Sikorski et al 1981). Stulberg et al (1984), however, found no significant relationship between the timing of ambulation and the formation of thrombi. The fact that the patients in this present series were allowed to walk as early as the third postoperative day may have contributed to the low incidence of deep vein thrombosis.

Some authorities believe that previous venous disease increases the risk of postoperative fresh deep vein thrombosis (Kakkar et al 1970; Cohen et al 1973), but others have found no such correlation (Barnes 1982; Francis et al 1983; Lotke et al 1984; Stulberg et al 1984). In our series, there was a total absence of previous venous disease, which might have contributed to the low incidence of deep vein thrombosis. 
It has been claimed that hyperlipidaemia may not only be associated with accelerated blood clotting but also with increase of blood viscosity and adhesiveness as well as aggregation of the red blood cells (Nicolaides and Irving 1975); the present study seems to support this view. None of our 244 patients had hyperlipidaemia; in fact, there is a low level of hyperlipidaemia in the Korean population.

The current prevailing opinion is that patients with thrombosis in the deep calf veins do not need to be treated with anticoagulants, and the present study supports this view.

The author wishes to thank Vana E. M. Kim, EdD, for reviewing and editing this manuscript.

No benefits in any form have been received or will be received from a commercial party related directly or indirectly to the subject of this article.

\section{REFERENCES}

Atik M, Harkess JW, Wichman H. Prevention of fatal pulmonary embolism. Surg Gynecol Obstet 1970; 130:403-13.

Barnes RW. Current status of noninvasive tests in the diagnosis of venous disease. Surg Clin North Am 1982; 62:489-500.

Clark C, Cotton LT. Blood-flow in deep veins of leg: recording technique and evaluation of methods to increase flow during operation. $B r J$ Surg 1968; 55:211-4.

Cohen SH, Ehrlich GE, Kaufiman MS, Cope C. Thrombophlebitis following knee surgery. J Bone Joint Surg [Am] 1973; 55-A: 106-12.

Doran FSA, Drury M, Sivyer A. A simple way to combat the venous stasis which occurs in the lower limbs during surgical operations. Br J Surg 1964; 51 :486-92.

Evarts CM, Feil EJ. Prevention of thromboembolic disease after elective surgery of the hip. J Bone Joint Surg [Am] 1971; 53A : $1271-80$.

Fahmy NR, Patel DG. Haemostatic changes and postoperative deepvein thrombosis associated with use of a pneumatic tourniquet. $J$ Bone Joint Surg [ Am] 1981; 63-A :461-5.

Francis CW, Marder VJ, Evarts CM, YaukoolbodiS. Two-step warfarin therapy: prevention of postoperative venous thrombosis without excessive bleeding. JAMA 1983; 249:374-8, 2021 (correction).

Gallus AS. Established venous thrombosis and pulmonary embolism. Clin Hematol 1981 ; 10:583-611.

Hull RD, Raskob GE. Current concepts review. Prophylaxis of venous thromboembolic disease following hip and knee surgery. $J$ Bone Joint Surg [ Am] 1986; 68-A : 146-50.

Jick H, Slone D, Westerholm B, et al. Venous thrombo-embolic disease and ABO blood type. A co-operative study. Lancet 1969; $1: 539-42$.
Kakkar VV, Howe CT, Nicolaides AN, Renney JTG, Clarke MB. Deep vein thrombosis of the leg: is there a "high risk" group? Am J Surg 1970; $120: 527-30$.

Kim Y-H, Suh J-S. Low incidence of deep vein thrombosis after cementless total hip replacement. J Bone Joint Surg [Am] 1988; 70-A :878-82.

Lewis CE Jr, Mueller C, Edward WS. Venous stasis on the operating table. Am J Surg 1972; 124:780-4.

Liu GC, Ferris EJ, Reifsteck JR, et al. Effect of anatomic variations on deep venous thrombosis of the lower extremity. Am J Roentgenol $1986 ; 146: 845-8$.

Lotke PA, Ecker ML, Alavi A, Berkowitz H. Indications for the treatment of deep venous thrombosis following total knee replacement. J Bone Joint Surg [Am] 1984; 66-A :202-8.

McKenna R, Bachmann F, Kaushal SP, Galante JO. Thromboembolic disease in patients undergoing total knee replacement. J Bone Joint Surg [ Am] 1976; 58-A :928-32.

McLachlin AD, McLachlin JA, Jory TA, Rawling EG. Venous stasis in the lower extremities. Ann Surg 1960; 152:678-85.

Morrey BF, Adams RA, Ilstrup DM, Bryan RS. Complications and mortality associated with bilateral or unilateral total knee arthroplasty. J Bone Joint Surg [Am] 1987; 69-A :484-8.

Nicolaides AN, Irving $D$. Clinical factors and the risk of deep vein thrombosis. In: Nicolaides AN, ed. Thromboembolism: aetiology, advances in prevention and management. Baltimore: University Park Press, 1975:193-204.

Nicolaides AN, Kakkar VV, Field ES, et al. Venous stasis and deep-vein thrombosis. Br J Surg 1972; 59:713-7.

Nillius AS, Nylander G. Deep vein thrombosis after total hip replacement: a clinical and phlebographic study. Br J Surg 1979; 66:324-6.

Salvati EA, Lachiewicz P. Thromboembolism following total hipreplacement arthroplasty: the efficacy of dextran-aspirin and dextran-warfarin in prophylaxis. J Bone Joint Surg [ Am] 1976; 58A :921-5.

Salzman EW, Harris WH. Prevention of venous thromboembolism in orthopaedic patients. J Bone Joint Surg [Am] 1976; 58-A :903-13.

Sevitt S, Gallagher N. Venous thrombosis and pulmonary embolism: a clinico-pathological study in injured and burned patients. $\mathrm{Br} J$ Surg 1961; 48:475-89.

Sikorski JM, Hampson WG, Staddon GE. The natural history and aetiology of deep vein thrombosis after total hip replacement. $J$ Bone Joint Surg [Br] 1981 ; 63-B:171-7.

Simon MA, Mass DP, Zarins CK, et al. The effect of a thigh tourniquet on the incidence of deep venous thrombosis after operations on the fore part of the foot. J Bone Joint Surg [Am] 1982; 64-A: 188-91.

Stulberg BN, Insall JN, Williams GW, Ghelman B. Deep-vein thrombosis following total knee replacement: an analysis of six hundred and thirty-eight arthroplasties. J Bone Joint Surg [Am] 1984 ; 66-A :194-201.

Stulberg BN, Lucas FV, Belhobek GH. Anti-thrombin III as a marker of venous thromboembolism: a preliminary report. Trans Orthop Res Soc 1984; 9:100.

Wright HP, Osborn SB, Edmonds DG. Effect of postoperative bed rest and early ambulation on the rate of venous blood-flow. Lancet $1951 ; 1: 22-5$ 